\title{
Threshold studies of the microwave instability in electron storage rings
}

\author{
K. L.F. Bane, Y. Cai, and G. Stupakov \\ SLAC National Accelerator Laboratory, Stanford University, Stanford, California 94309, USA
}

(Received 12 July 2010; published 7 October 2010)

\begin{abstract}
We use a Vlasov-Fokker-Planck program and a linearized Vlasov solver to study the microwave instability threshold of impedance models: (1) a $Q=1$ resonator and (2) shielded coherent synchrotron radiation (CSR), and find the results of the two programs agree well. For shielded CSR we show that only two dimensionless parameters, the shielding parameter $\Pi$ and the strength parameter $S_{\mathrm{csr}}$, are needed to describe the system. We further show that there is a strong instability associated with CSR, and that the threshold, to good approximation, is given by $\left(S_{\mathrm{csr}}\right)_{\mathrm{th}}=0.5+0.12 \Pi$. In particular, this means that shielding has little effect in stabilizing the beam for $\Pi \lesssim 2$; for larger $\Pi$ it is effective, with threshold current depending on shielding aperture as $h^{-3 / 2}$. We, in addition, find another instability in the vicinity of $\Pi=0.7$ with a lower threshold, $\left(S_{\mathrm{csr}}\right)_{\mathrm{th}} \approx 0.2$. We find that the threshold to this instability depends strongly on damping time, $\left(S_{\mathrm{csr}}\right)_{\mathrm{th}} \sim \tau_{p}^{-1 / 2}$, and that the tune spread at threshold is small—both hallmarks of a weak instability.
\end{abstract}

DOI: 10.1103/PhysRevSTAB.13.104402

PACS numbers: 29.27. $-\mathrm{a}, 41.75 . \mathrm{Ht}$

\section{INTRODUCTION}

In the design of electron storage rings there is often a need to evaluate the threshold to the (longitudinal) microwave instability. Several computational tools are available that allow us, once given the wakefield representing a ring, to numerically find the threshold current and to simulate the development of the instability. In this work, we present results of computer simulations using two codes recently developed at the SLAC National Accelerator Laboratory: a Vlasov-Fokker-Planck (VFP) solver based on an algorithm by Warnock and Ellison [1], and a program that finds the threshold from the linearized Vlasov equation.

We apply the programs to finding the instability threshold for two models of ring impedance: (1) the $Q=1$ resonator and (2) shielded coherent synchrotron radiation (CSR). The former model has often been used to represent the impedance of a storage ring (see e.g. [2,3]), while the latter one can describe the dominant effect in rings with short bunches. The $Q=1$ model has a well-behaved wake function, whereas the CSR wake is singular and requires special care for its use in simulation.

The microwave instability is normally a strong instability with a fast growth rate, whose threshold can roughly be described by the Boussard criterion [4]. Fifteen years ago Oide [5], and then others [6], found that, under certain conditions, a different type, so-called weak instability, is also possible. These authors showed that a resistive impedance, one that generates an asymmetric bunch shape and results in negligible tune spread within the beam, can be the source of the weak instability. Unlike with the strong instability, the threshold to the weak instability is sensitive to the synchrotron radiation damping time, $\tau_{d}$; for a pure resistive impedance the threshold current varies as $\tau_{d}^{-1 / 2}$. The current Stanford Linear Collider (SLC) damping ring, according to analysis, is an example of a ring that exhibits a weak instability [7].

In this paper, we begin by describing the VFP and the Vlasov codes that will be used for the simulations. Then, for the two impedance models, we give and compare simulation results of the two programs. In the VFP calculations we also vary the damping parameter, representing synchrotron radiation damping, to spot check for instability type (strong vs weak). Note that numerical studies of the threshold of a $Q=1$ resonator wake have been performed before by Oide and Yokoya [8], and others [9-11], but the systematic study of the microwave instability driven by shielded CSR is new.

In this paper we assume the slippage factor $\eta$ is always positive. We work in Gaussian units.

\section{VLASOV-FOKKER-PLANCK SOLVER}

Let us consider the longitudinal motion of a bunched beam in an electron storage ring. When there is a collective force induced by the bunch distribution $\lambda(\theta, q)$ through the wakefield $w(q)$, the evolution of the beam density distribution (in longitudinal phase space) $\psi(\theta, q, p)$ is governed by the Vlasov-Fokker-Planck equation,

$$
\frac{\partial \psi}{\partial \theta}-\{H, \psi\}=2 \beta \frac{\partial}{\partial p}\left(p \psi+\frac{\partial \psi}{\partial p}\right),
$$

where the Poisson bracket is defined by $\{f, g\}=\left(\frac{\partial f}{\partial q} \frac{\partial g}{\partial p}-\right.$ $\left.\frac{\partial f}{\partial p} \frac{\partial g}{\partial q}\right)$. The Hamiltonian

$$
\begin{aligned}
H(\theta, q, p)= & \frac{1}{2}\left(q^{2}+p^{2}\right)-I \int_{-\infty}^{q} d q^{\prime \prime} \int_{-\infty}^{\infty} d q^{\prime} \lambda\left(\theta, q^{\prime}\right) \\
& \times w\left(q^{\prime \prime}-q^{\prime}\right)
\end{aligned}
$$

with $\lambda(\theta, q)=\int_{-\infty}^{\infty} \psi(\theta, q, p) d p$. The independent vari- 
able $\theta=\omega_{s} t$, with $\omega_{s}$ the (nominal) synchrotron frequency and $t$ the time. We use a normalized coordinate system: $q=z / \sigma_{z 0}$, where $z$ is longitudinal position, with the positive $z$ axis pointing to the front (the direction of motion), and $\sigma_{z 0}$ is nominal (zero current) rms bunch length; $p=-\delta / \sigma_{\delta 0}$, where $\delta$ is relative energy deviation and $\sigma_{\delta 0}$ is nominal energy spread. We define normalized current as

$$
I=\frac{r_{e} N_{b}}{2 \pi \nu_{s 0} \gamma \sigma_{\delta 0}}
$$

with $r_{e}$ is the classical electron radius, $N_{b}$ the bunch population, $\nu_{s 0}$ the nominal synchrotron tune, and $\gamma$ the Lorentz energy factor; $I$ has dimensions of length. The terms on the right of Eq. (1) are the synchrotron radiation damping and quantum diffusion terms; here $\beta=1 / \omega_{s} \tau_{d}$, with $\tau_{d}$ the longitudinal damping time. Note that the wake $w(\Delta q)$-with dimension of inverse length - represents the effect of the entire ring, that argument $\Delta q>0$ implies the test particle is ahead of the driving charge, and that $w>0$ indicates energy loss.

To solve Eq. (1), Warnock and Ellison developed a robust algorithm based on the Perron-Frobenius operator, with the solution obtained on a regular grid in longitudinal phase space [1]. We have rewritten their code in $\mathrm{C}++$, making some modifications to their grid interpolation scheme, in order to improve detection of the threshold to instability. In a typical run for this report, the maximum of $|q|$ and $|p|$ is 8, with 300 mesh points in each direction; the number of time steps per synchrotron period is 1024, with a total run lasting $N_{s}=400$ synchrotron periods. The damping parameter is nominally taken to be small, $\beta=1.25 \times$ $10^{-3}$; to check for instability type (strong vs weak) we perform extra runs with $\beta=2.5 \times 10^{-3}$.

The program initializes $\lambda$ with the solution to the Haïssinski equation [12], and the energy distribution with the nominal Gaussian distribution. To find the threshold we perform several runs at different currents. If after a short transient interval (typically of $N_{s} \sim 100$ ) the rms of the energy parameter, $\sigma_{p}$, damps down to almost exactly the nominal value of 1 , we consider the current to be below threshold, else it is above threshold. We take as threshold the current at which $\sigma_{p}$ begins to deviate from the nominal value of 1 .

\section{LINEARIZED VLASOV (LV) CODE}

The approach described in the previous section solves a full VFP problem and for an unstable equilibrium allows one to find the threshold to the instability as well as the nonlinear evolution of beam phase space above threshold. In cases where we only want to know the threshold current, a linearized Vlasov (LV) analysis can be used. We have developed a computer code that numerically solves the LV problem. The method for finding the threshold to instability in this case begins by finding the equilibrium density distribution, $\psi_{0}(q, p)$, through the solution of the Haïssinski equation. We linearize the Vlasov equation about this distribution, taking $\psi(\theta, q, p)=\psi_{0}(q, p)+$ $\psi_{1}(\theta, q, p)$, and assuming that $\left|\psi_{1}\right| \ll\left|\psi_{0}\right|$. The linearized Vlasov equation takes the form

$$
\begin{aligned}
& \frac{\partial \psi_{1}}{\partial \theta}+p \frac{\partial \psi_{1}}{\partial q}-K_{0}(q) \frac{\partial \psi_{1}}{\partial p}-K_{1}(\theta, q) \frac{\partial \psi_{0}}{\partial p}=0, \\
& K_{0}(q)=q-I \int_{-\infty}^{\infty} d q^{\prime} d p \psi_{0}\left(q^{\prime}, p\right) w\left(q-q^{\prime}\right), \\
& K_{1}(\theta, q)=-I \int_{-\infty}^{\infty} d q^{\prime} d p \psi_{1}\left(\theta, q^{\prime}, p\right) w\left(q-q^{\prime}\right) .
\end{aligned}
$$

Equation (4) is solved numerically on a mesh in $q-p$ space, starting from a randomly generated initial distribution function $\psi_{1}$. Typically the mesh, in each direction, spans \pm 6 and contains $\sim 250-300$ points. If the system is unstable, after a sufficient time the evolution becomes dominated by the fastest exponentially growing mode. The growth rate $\Gamma$ of the instability is found numerically by fitting $e^{\Gamma \theta}$ to the time evolution of the system. The code also computes the phase portrait of the unstable mode. Typically we take 100 time steps per synchrotron period.

The numerical algorithm is implemented in MATHEMATICA. The wake function in the Vlasov equation can be input as an arbitrary MATHEMATICA function, and includes predefined resistive, inductive, and CSR wakes. The code was tested on an SLC damping ring wake [7], and the results compared well with results of other programs [13].

\section{CALCULATIONS}

\section{A. $Q=1$ resonator wake}

The $Q=1$ resonator wake has often been used to model the impedance of a ring. The resonator wake is nonzero only for negative argument (the test particle behind the driving charge); it is given by

$w(q)=W H(-q) e^{\Omega q / 2}\left[\cos \left(\frac{\sqrt{3} \Omega q}{2}\right)+\frac{1}{\sqrt{3}} \sin \left(\frac{\sqrt{3} \Omega q}{2}\right)\right]$,

with parameters amplitude $W$ (of dimension inverse length) and $\Omega \equiv \omega_{r} \sigma_{z 0} / c$, with $\omega_{r}$ the resonator frequency; here $H(x)$ is the unit step function $[H(x)=1$ for $x>0,=0$ for $x<0]$.

We have performed stability calculations for this model, with $\Omega$ over the range $[0.25,2.0]$. Analysis shows that the dynamics described by the Vlasov equation in this case, and hence the threshold of the instability, depend only on two dimensionless parameters, $\Omega$ and $S_{\text {res }}=I W$ [8]. In Fig. 1 we plot the resulting threshold value of $S_{\text {res }}$ vs $\Omega$, where the VFP results are given by blue circles (that are joined by straight lines), those of LV by red squares (an error bar indicates uncertainty in result). In general, we see 


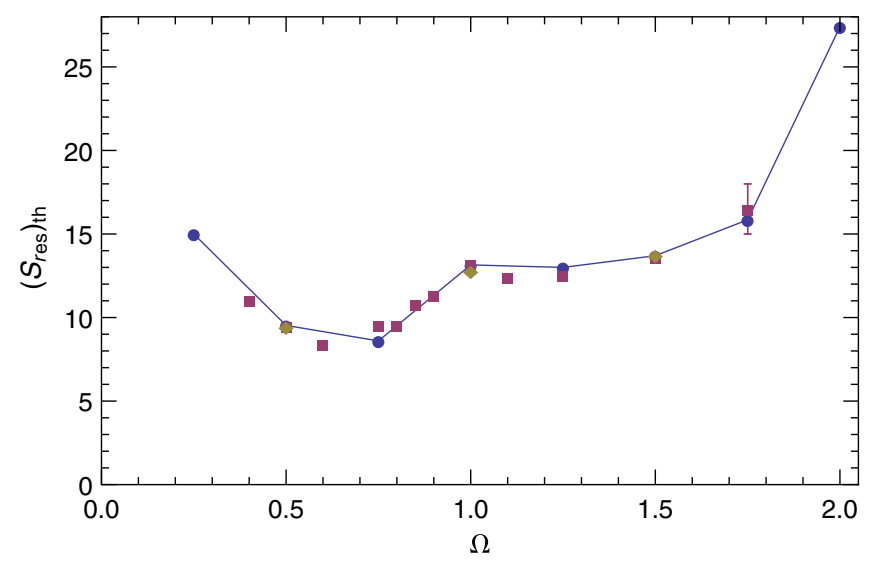

FIG. 1. For the $Q=1$ resonator wake, threshold value of $S_{\text {res }}$ vs parameter $\Omega$. Symbols give results of the VFP solver (blue circles), the LV code (red squares), and the VFP solver with twice the damping parameter: $\beta=2.5 \times 10^{-3}$ (olive diamonds).

good agreement between the results of the two methods, and also reasonably good agreement when compared to the earlier results of Oide and Yokoya and others [8-11]. In Fig. 1 , at $\Omega=0.5,1.0,1.5$, we also plot the VFP results for the case where the synchrotron radiation damping rate is increased by a factor of 2 (the olive diamonds). We see that the threshold is not sensitive to the damping rate, indicating that the instability of the $Q=1$ resonator is of the usual, strong type. In Fig. 2 we present the Haïssinski solution at threshold for selected values of $\Omega$.

\section{B. CSR wake}

We consider the CSR wakefield generated by an electron moving on a circular orbit of radius $\rho$ in the middle of two (perfectly conducting) parallel plates that are separated by a distance $2 h$. In the case of no shielding $(h \rightarrow \infty)$ the wake is nonzero only for positive $q$ (i.e. the test particle ahead of

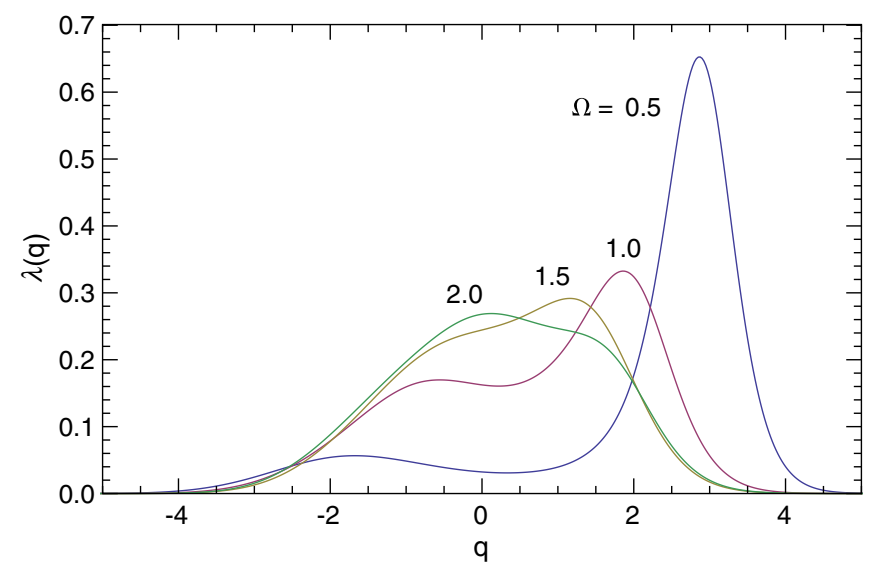

FIG. 2. For the $Q=1$ resonator, the Haïssinski solution at threshold, for the cases $\Omega=0.5,1.0,1.5,2.0$. Note that the bunch head is to the right. the driving charge), and given by

$$
w_{0}(q)=-\frac{4 \pi}{3^{4 / 3}} H(q) \frac{\rho^{1 / 3}}{\left(q \sigma_{z 0}\right)^{4 / 3}} .
$$

This wake is singular and requires special care in its use. In the simulations, we obtain the bunch wake $v_{\text {ind }}$ by convolving with the bunch shape $\lambda$. For such a singular wake, however, we integrate by parts and discard the boundary term; i.e., we let the bunch wake $v_{\text {ind }}(q)=\int s\left(q^{\prime}\right) \lambda^{\prime}(q-$ $\left.q^{\prime}\right) d q^{\prime}$, where $s(q)=\int_{-\infty}^{q} w\left(q^{\prime}\right) d q^{\prime}$ and $\lambda^{\prime}$ is the derivative of the bunch distribution (for a justification, see, e.g., Ref. [14]). Because of the $\lambda^{\prime}$ in the integral, simulation with such a wake is more sensitive to numerical errors or noise, and obtaining reliable results is more of a challenge.

With shielding, the wake $w(q)=w_{0}(q)+w_{1}(q)$, with [15]

$$
w_{1}(q)=-\rho^{1 / 3}\left(\frac{\Pi}{\sigma_{z 0}}\right)^{4 / 3} G(\Pi q),
$$

where the shielding parameter $\Pi=\sigma_{z 0} \rho^{1 / 2} / h^{3 / 2}$, and $2 h$ is the separation between the two plates. The term $w_{1}(q)$ is the contribution to the wake of the image charges generated by the metal plates; note that it is in general nonzero for both signs of argument. The function $G$ is given by

$$
G(\zeta)=8 \pi \sum_{k=1}^{\infty} \frac{(-1)^{k+1}}{k^{2}} \frac{Y_{k}(\zeta)\left[3-Y_{k}(\zeta)\right]}{\left[1+Y_{k}(\zeta)\right]^{3}},
$$

where $Y_{k}$ is a root of the equation

$$
Y_{k}-\frac{3 \zeta}{k^{3 / 2}} Y_{k}^{1 / 4}-3=0 .
$$

This equation has two real, positive roots and two complex roots. We choose the smaller real root when $\zeta<0$, the larger real root when $\zeta>0$. Normally, in the simulations, we sum $k$ up to 25 .

It can be seen from Eqs. (6) and (7), that for the CSR wake the beam dynamics depend only on two dimension-

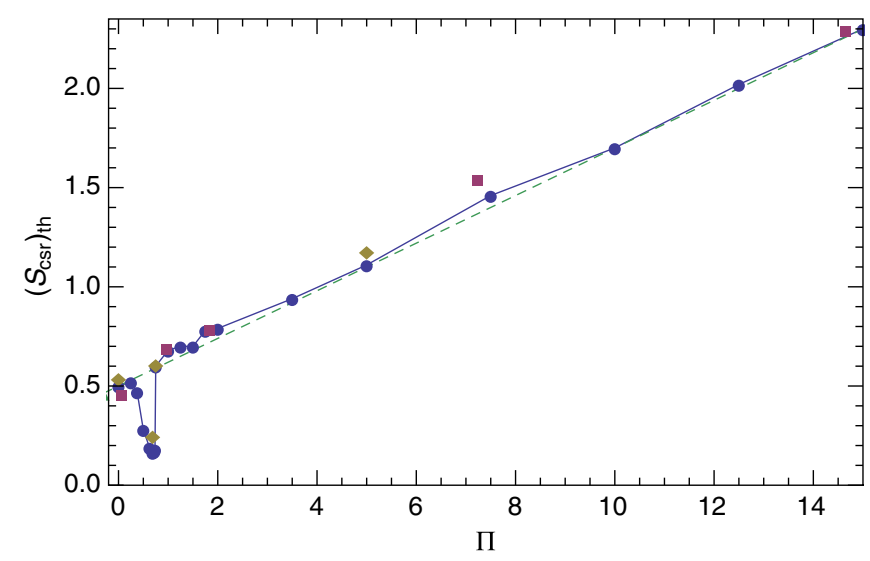

FIG. 3. For the CSR wake, threshold value of $S_{\text {csr }}$ vs shielding parameter, $\Pi=\rho^{1 / 2} \sigma_{z 0} / h^{3 / 2}$. Symbols give results of the VFP solver (blue circles), the LV code (red squares), and the VFP solver with twice stronger radiation damping (olive diamonds). 

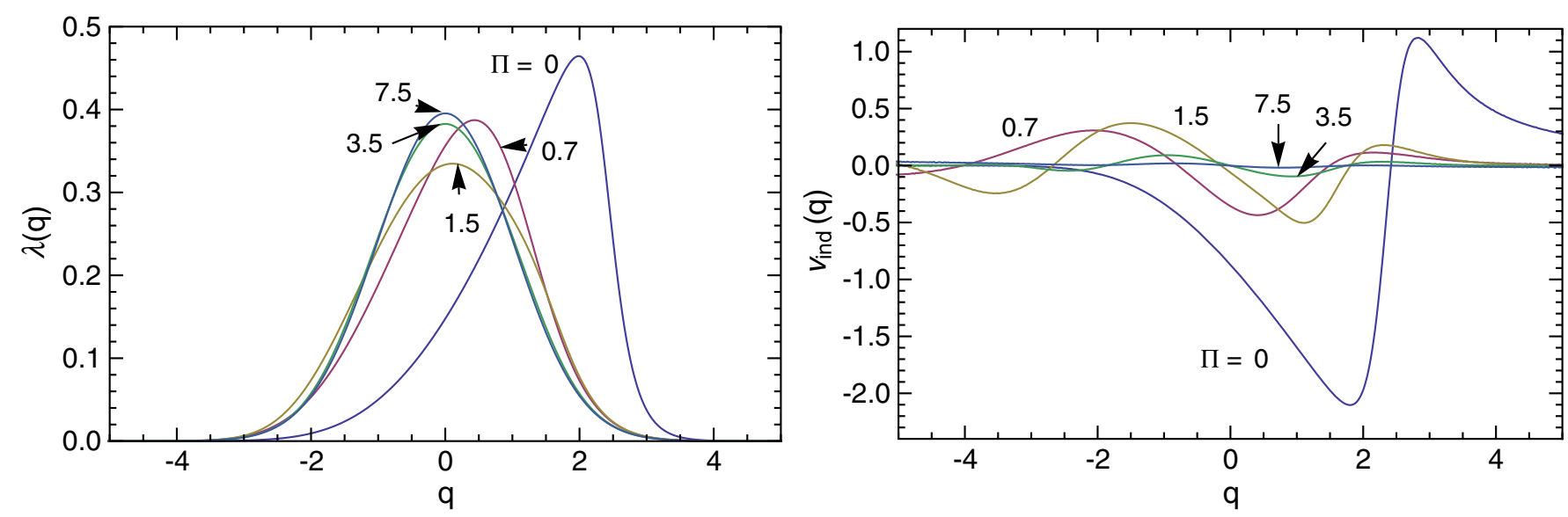

FIG. 4. Haïssinski solution at threshold and wake induced voltage for shielded CSR, for the cases $\Pi=0.0,0.7$, 1.5, 3.5, 7.5. Note that the bunch head is to the right.

less parameters, the shielding parameter $\Pi$ and the strength parameter $S_{\mathrm{csr}}=I \rho^{1 / 3} / \sigma_{z 0}^{4 / 3}$.

We have performed stability calculations for this model, for shielding parameter $\Pi$ up to 15 . Our results of threshold strength parameter $\left(S_{\mathrm{csr}}\right)_{\text {th }}$ vs $\Pi$ are plotted in Fig. 3. Symbols give results of the VFP solver (blue circles, connected by straight lines), the LV code (red squares), and the VFP solver with twice stronger radiation damping (olive diamonds). In general, we find good agreement between the VFP and LV results. With no shielding $\left(S_{\mathrm{csr}}\right)_{\text {th }}=0.50$. The rest of the results (except for those in a dip region in the vicinity of $\Pi=0.7$ ) closely follow the straight line $\left(S_{\mathrm{csr}}\right)_{\mathrm{th}}=0.5+0.12 \Pi$ (the dashes). In particular, this result means that shielding has little effect in stabilizing the beam for $\Pi \lesssim 2$; for larger $\Pi$, however, it is effective, with threshold current depending on plate spacing as $h^{-3 / 2}$.

In Fig. 3 we also see that the nominal and stronger damping VFP results agree well (except in the dip region), indicating that the instability is of the strong type. In Fig. 4 we plot the Haiissinski solution at threshold and the wake induced voltage $v_{\text {ind }}$ for selected values of shielding parameter, $\Pi$. With no shielding the bunch shape is markedly triangular; with increasing shielding it moves gradually toward that of the unperturbed Gaussian. We see that $v_{\text {ind }}$, in amplitude, drops quickly as $\Pi$ increases from zero; by $\Pi \gtrsim 1.5$ this function, in addition, has become largely inductive.

\section{Weak instability}

At $\Pi=0.7$, in the dip of Fig. 3, the VFP threshold is $\left(S_{\mathrm{csr}}\right)_{\mathrm{th}}=0.17$ for nominal damping $\left(\beta=1.25 \times 10^{-3}\right)$, 0.25 for twice stronger damping, and 0.12 for twice weaker damping (result not plotted). These results are consistent with a threshold damping time dependence of $\beta^{1 / 2}$, indicating that, in the region of the dip, the instability is of the weak type. Note that with the LV formulation we did not obtain a reliable result in the dip region.
To focus in on the dip region, we plot in Fig. 5 the value of $\sigma_{p}$ averaged over the first 400 synchrotron periods, $\left\langle\sigma_{p}\right\rangle$, vs $S_{\text {csr }}$ as given by the VFP simulations (at $\Pi=$ 0.7 ), both for nominal damping (the blue circles) and for twice stronger damping (olive diamonds). We see that there are actually two thresholds to instability at this value of $\Pi$ : The first (at $S_{\mathrm{csr}}=0.17$ or 0.25 ) depends strongly on radiation damping time, and thus concerns a weak type of instability. A second instability threshold (at $S_{\mathrm{csr}}=$ 0.62 , which incidentally lies close to the straight line of Fig. 3) is insensitive to damping time, and concerns a strong instability. In between the two (for $S_{\mathrm{csr}}=$ $0.54-0.62$ ) there is a region of no instability. In more detail, in Fig. 6 we plot (for $\Pi=0.7$ and nominal damping) $\sigma_{p}$ over the 400 synchrotron periods of simulation, for $S_{\mathrm{csr}}=$ 0.23 and 0.54 . The former case is clearly above threshold. For the latter case, however, we see that after an initial



FIG. 5. For shielded CSR with $\Pi=0.7$, the value of $\sigma_{p}$, averaged over 400 synchrotron periods, $\left\langle\sigma_{p}\right\rangle$, as given by the VFP simulations. The case of nominal damping $\beta=1.25 \times$ $10^{-3}$ is given by blue circles (connected by straight lines), the case of $\beta=2.5 \times 10^{-3}$ by olive diamonds. 

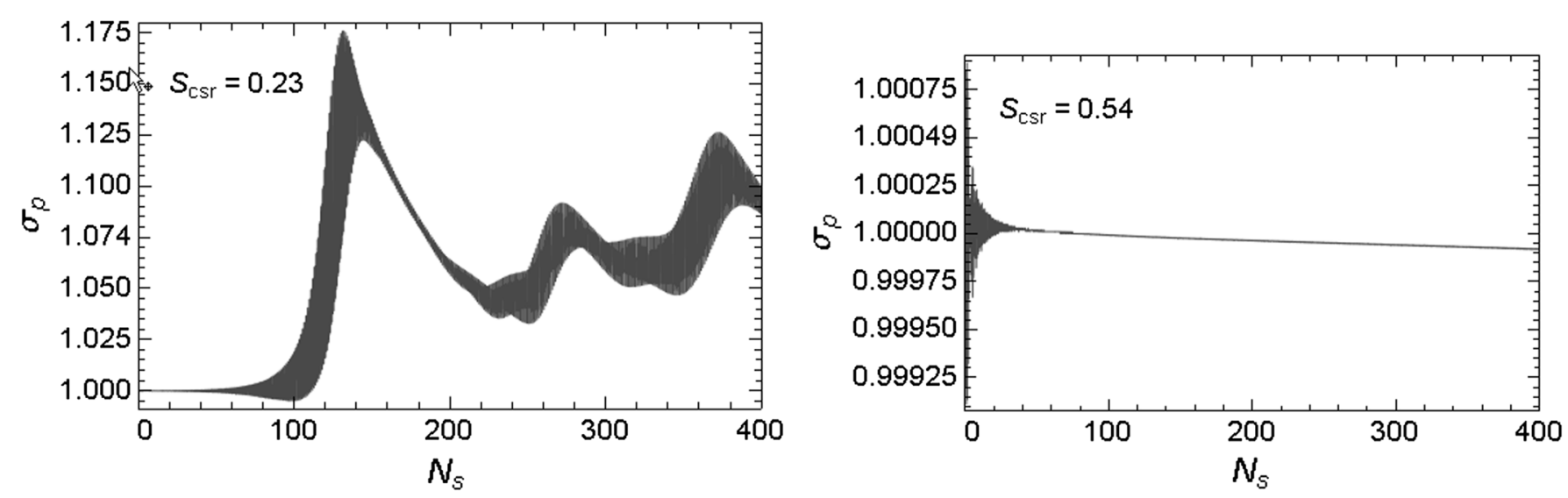

FIG. 6. For shielded CSR with $\Pi=0.7$ and nominal damping, rms of $p$ coordinate, $\sigma_{p}$, vs synchrotron period number, $N_{s}$, for cases $S_{\mathrm{csr}}=0.23$ and 0.54 .

(small) transient, $\sigma_{p}$ remains very close to 1 ; i.e., the beam appears to be quiet and stable.

From these results we can see that the behavior of the beam below the strong instability threshold (below $S_{\mathrm{csr}}=$ 0.62 ) depends on the synchrotron radiation damping rate. Another way for a weak instability to be suppressed is by introducing incoherent tune spread in the beam (by distorting the potential well) to Landau damp it [5]. We expect our model can apply to storage rings where shielded CSR is the dominant impedance. However, in a real machine there are other sources of impedance, and since it only takes a small amount of tune spread to suppress a weak instability $[5,7]$, even in a machine that is dominated by shielded CSR the weak instability may not be observed.

A weak instability should only appear when there is a small tune spread. To test this with the CSR wake we compute the incoherent tune, given by

$$
\nu_{s}(J)=\nu_{s 0}\left[\frac{1}{\sqrt{2} \pi} \int_{q_{b}}^{q_{e}} \frac{d q}{\sqrt{u\left(q_{b}\right)-u(q)}}\right]^{-1}
$$

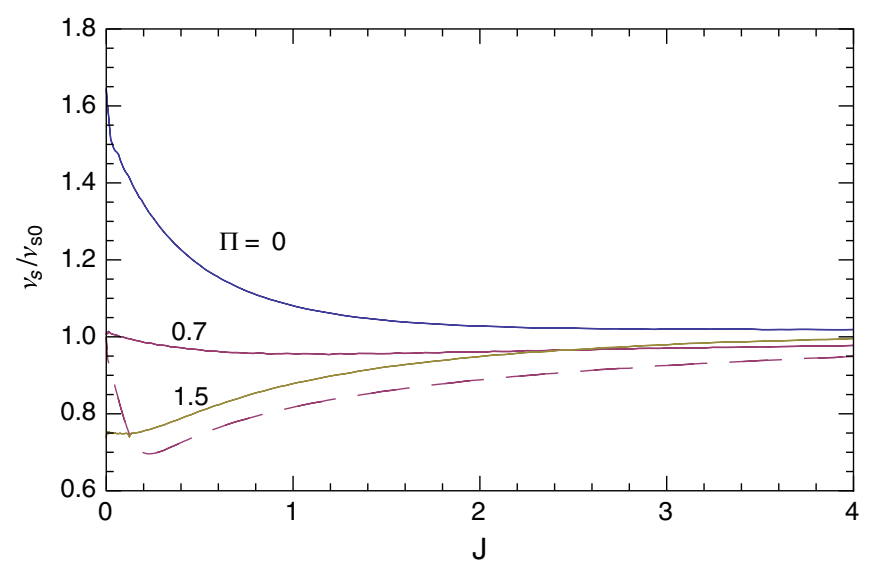

FIG. 7. Shielded CSR: incoherent tune at threshold due to the distorted potential well, as a function of action, $J$, for cases $\Pi=$ $0,0.7$, and 1.5. The dashed curve is explained in the text below. with $J$ the action. Here the potential well energy of the Haïssinski solution $u(q)=-\ln \lambda(q) ; q_{b}$ and $q_{e}$ are positions on opposite sides of the minimum of $u$, with $u\left(q_{b}\right)=$ $u\left(q_{e}\right)$; and $J=u\left(q_{b}\right)-\min (u)$. In Fig. 7 we plot $\nu_{s}$ at threshold for cases $\Pi=0,0.7$, and 1.5 (the solid curves). The rms of the tune-normalized by $\nu_{s 0}$ and weighted by the bunch distribution $-\sigma_{\nu} / \nu_{s 0}=0.19,0.02,0.06$, for the cases $\Pi=0,0.7,1.5$, respectively. We see that the case $\Pi=0.7$, the case that experienced a weak instability, also has the smallest (by far) tune spread of the three. This result thus, at least qualitatively, supports our expectation that a weak instability is consistent with a small tune spread. Further quantification of the connection between tune spread and instability strength is beyond the scope of this report.

Finally, how can we understand the phenomenon shown in Fig. 5 of first a weak instability and then a strong instability, with a quiet region in between? To try to answer this question we have also calculated the incoherent tune for $\Pi=0.7$ and $S_{\mathrm{csr}}=0.54$ (at the beginning of the quiet region). The result is shown in Fig. 7, the dashed curve. Here the rms tune spread $\sigma_{\nu} / \nu_{s 0}=0.09$, which is quite large compared to what it was at threshold, at $S_{\mathrm{csr}}=0.17$ (compare the red dashed curve with the red solid curve in Fig. 7). So our explanation of the phenomenon of Fig. 5 is: at threshold to the weak instability there is little tune spread; as the current increases, both the instability growth rate and tune spread increase; eventually, for a current in the quiet region, we believe that the tune spread overpowers the instability and completely suppresses it.

\section{CONCLUSION}

For the task of finding the microwave threshold, we have shown that our Vlasov-Fokker-Planck and linearized Vlasov solvers agree quite well when applied to impedance models of (1) a $Q=1$ resonator and (2) the more difficult 
to compute CSR wake between two parallel plates. For shielded CSR we have shown that only two dimensionless parameters, the shielding parameter $\Pi$ and the strength parameter $S_{\mathrm{csr}}$ are needed to describe the system. We have, in addition, shown that there is a strong instability associated with CSR, one insensitive to the synchrotron radiation damping time, and that its threshold is, to good approximation, given by $\left(S_{\mathrm{csr}}\right)_{\mathrm{th}}=0.5+0.12 \Pi$. In particular, this means that shielding has little effect in stabilizing the beam for $\Pi \lesssim 2$; however, for larger $\Pi$ it is effective, with threshold current depending on plate spacing as $h^{-3 / 2}$.

For shielded CSR we have, in addition, found a weak instability, located in the vicinity of $\Pi=0.7$, where the threshold drops to $\left(S_{\text {csr }}\right)_{\text {th }} \approx 0.2$. With VPF simulations we have demonstrated that the threshold to this instability is sensitive to synchrotron radiation damping time $\tau_{p}$, varying as $\left(S_{\mathrm{csr}}\right)_{\mathrm{th}} \sim \tau_{p}^{-1 / 2}$, and that the tune spread at threshold is small-both hallmarks of a weak instability. In our VFP simulations at $\Pi=0.7$ we have found a weak instability starting at $S_{\mathrm{csr}} \approx 0.2$, a strong instability beginning at $S_{\mathrm{csr}}=0.6$, and a stable region in between the two.

\section{ACKNOWLEDGMENTS}

This work was supported by Department of Energy Contract No. DEAC0276SF00515.
[1] R. Warnock and J. Ellison, Report No. SLAC-PUB-8404, 2000.

[2] A. Hofmann and J.R. Maidment, CERN, LEP-Note Report No. 168, 1979.

[3] Y. Cai et al., Phys. Rev. ST Accel. Beams 12, 061002 (2009).

[4] D. Boussard, CERN/PS-BI, 1972.

[5] K. Oide, KEK Report No. 94-138, 1994.

[6] A. Chao et al., in Proceedings of the Particle Accelerator Conference, Dallas, TX, 1995 (IEEE, New York, 1995), p. 3040.

[7] K. Bane and K. Oide, in Proceedings of the Particle Accelerator Conference, Dallas, TX, 1995 (Ref. [6]), p. 3105 .

[8] K. Oide and K. Yokoya, KEK, Report No. 90-10, 1990.

[9] K. Bane, S. Heifets, and G. Stupakov, at the ILCDR06 Workshop, Cornell, 2006.

[10] S. Heifets, Report No. SLAC-PUB-12122, 2006.

[11] NSLSII Conceptual Design Report at http://www.bnl.gov/ nsls2/project/CDR, Sec. 6.2.3.2.

[12] J. Haïssinski, Nuovo Cimento B 18, 72 (1973).

[13] R. L. Warnock and J. A. Ellison, in The Physics of High Brightness Beams (World Scientific, Singapore, 2000), pp. 322-348.

[14] G. Stupakov, lecture notes at http://www.slac.stanford .edu/ stupakov/uspas07/lectures.pdf.

[15] J. B. Murphy, S. Krinsky, and R.L. Gluckstern, Part. Accel. 57, 9 (1997). 